\title{
RUDJER BOŠKOVIĆ INSTITUTE RADIOCARBON MEASUREMENTS XII
}

DUŠAN SRDOČ, NADA HORVATINČIĆ, INES KRAJCAR BRONIĆ, BOGOMIL OBELIĆ

Rudjer Bošković Institute, P. O. Box 1016, 41001 Zagreb, Croatia

and

ADELA SLIEPČEVIĆ

Faculty of Veterinary Medicine, University of Zagreb

\section{INTRODUCTION}

We present here radiocarbon analyses made since our previous list (Srdoč et al. 1989). Sample pretreatment, combustion and counting techniques are essentially the same as described previously (Srdoč, Breyer \& Sliepčević 1971), supplemented by new techniques for groundwater processing (Srdoč et al. 1979). The calculation of ages follows conventional protocol (Stuiver \& Polach 1977). These ages can be converted from the 5570-year half-life to the chronometrically more correct halflife of 5730 years by multiplying by the factor, 1.029. Ages and standard deviations of all samples are adjusted for stable isotope fractionation according to the recommendations in Stuiver and Polach (1977), except for groundwater, calcareous deposits and aquatic plants. The $\delta^{13} \mathrm{C}$ values of the latter reflect the environmental conditions during their formation or growth rather than fractionation (Mook 1976; Krajcar Bronić et al. 1986; Marčenko et al. 1989). Thus, any percent modern (pMC) correction based on $\delta^{13} \mathrm{C}$ values is meaningless or even misleading. Sample descriptions are prepared in collaboration with collectors and submitters. Calibrated ages were calculated using the program of Stuiver and Reimer (1987).

\section{ACKNOWLEDGMENTS}

We thank E. Hernaus for preparation of samples and methane synthesis, A. Turković for data processing and $\mathrm{P}$. Hojski for technical assistance.

\section{ARCHAEOLOGICAL SAMPLES}

YUGOSLAVIA

\section{Z-1966. Statue of Christ's head}

$98.9 \pm 0.8 \mathrm{pMC}$

Sample is from a wooden statue of Christ's head (43 tree rings), from a private collection. Collected and submitted 1987 by V. Lavrenčič, Ljubljana, Slovenia.

Comment: Calibrated range: AD 1686-1955.

\section{Vela spilja series}

Charcoal mixed with clay from Vela spilja cave, Korčula Island, $0.85-1.64 \mathrm{~m}$ below ground level, near Vela Luka ( $\left.48^{\circ} 58^{\prime} \mathrm{N}, 16^{\circ} 44^{\prime} \mathrm{E}\right), 130 \mathrm{~m}$ asl, Croatia. Collected and submitted Dec. 1987 by B. Čečuk, Archaeological Institute, Yugoslav Academy of Sciences and Arts, Zagreb.

Z-1968.

$6990 \pm 120$

Charcoal from Layer 5 above a grave, $0.85-1.12 \mathrm{~m}$ depth. 
Z-1967.

Charcoal from Layer 6 above the same grave, 1.12-1.64 m depth.

Comment (B.Č.): This was a systematic archaeological investigation of a Neolithic site. The expected age was $\sim 6000 \mathrm{BP}$. The date from an earlier measurement was Z-1742: $5430 \pm 100$ (Srdoč et al. 1989: 86).

\section{Ajdovska jama cave series}

Charcoal from Ajdovska jama cave at Nemška Vas near Krško $\left(45^{\circ} 58^{\prime} \mathrm{N}, 15^{\circ} 30^{\prime} \mathrm{E}\right)$, east Slovenia. Collected by M. Horvat, Faculty of Sciences, Ljubljana and submitted 1987 by A. Šercelj, Slovenian Academy of Sciences and Arts, Ljubljana. Samples from a systematic excavation of a Neolithic site. Dates correspond to earlier measurements, Z-1042 to -1045 (Srdoč et al. 1984: 451), Z-1178, -1179, -1554, -1602, -1603 (Srdoč et al. 1987: 138-139) and Z-1822, -1860 (Srdoč et al. 1989: 86).

\section{Z-2042. Ajdovska jama 41/87}

$5230 \pm 110$

Charcoal from Quadrant 35. Sample was associated with human bones.

Z-2043. Ajdovska jama 34/87

$4820 \pm 100$

Charcoal from a brown loose layer, Quadrant 35.

Z-2044. Ajdovska jama 18/87

$4900 \pm 100$

Charcoal from a gray ash layer, Quadrant 35.

\section{Z-2123. Ajdovska jama 42}

$5360 \pm 130$

Charcoal with charred pollen from a central hall, Quadrant 24, Section 12.

\section{Z-2179. Ajdovska jama 19}

Charcoal with charred pollen from Quadrant 39, Section 68.

Comment (D.S.): Z-1603 is an outlier in this series; Z-1822 is a speleothem whose age agrees well with the charcoal age, if we assume the initial ${ }^{14} \mathrm{C}$ activity, $\mathrm{A}_{\mathrm{o}}$, of speleothems equal to $85 \mathrm{pMC}$, a pervasive value for $A_{0}$ in karst of Dinarides.

\section{Sisak series}

Fragments of a charred beam from a building near the Kupa River bank at Sisak $\left(45^{\circ} 30^{\prime} \mathrm{N}\right.$, $16^{\circ} 20^{\prime} \mathrm{E}$ ), Croatia. Collected and submitted 1988 by V. Nenadić, Department of Archaeology, Institute of Historical Sciences, University of Zagreb.

\section{Z-2063. Sisak 1}

$170 \pm 110$

Fragment of a charred beam, $3.85 \mathrm{~m}$ depth.

Z-2064. Sisak 2

$20 \pm 110$

Fragment of a charred beam, $4.10 \mathrm{~m}$ depth. 
Comments: (V.N.): Expected age: 250 BP. (D.S.): The expected age is within calibrated range (AD 1675-1955), according to Stuiver and Reimer (1987).

Z-2112. Sisak

$1990 \pm 120$

Fragment of a wooden beam buried in the Kupa River bed, probably from the foundation of a Roman mint. Collected and submitted 1989 by B. Slovenec and G. Grgić, Sisak.

Z-2065. Podsreda

$600 \pm 80$

Remnants of a rotten wooden beam built into the south facade of a stone wall in Podsreda $\left(46^{\circ} 2^{\prime} \mathrm{N}\right.$, $15^{\circ} 38^{\prime} \mathrm{E}$ ), near Bistrica ob Sotli. Collected and submitted 1988 by B. Uršić, Regional Institute for Protection of Cultural Monuments, Zagreb.

Comment (B.U.): Expected age: Middle Ages (12th or 13th century).

Z-2096. Vladikina ploča

$2650 \pm 110$

Charcoal from a fireplace in Vladikina ploča cave, Rsovac near Pirot $\left(43^{\circ} 11^{\prime} 26^{\prime \prime} \mathrm{N}, 22^{\circ} 45^{\prime} 17^{\prime \prime} \mathrm{E}\right)$, $758 \mathrm{~m}$ asl, $20 \mathrm{~m}$ from the entrance, covered with 1 to $5-\mathrm{cm}$-thick calcite flowstone. Collected and submitted 1988 by D. Gavrilović, University of Belgrade.

Comment (D.G.): Expected age: 500 BP. Dates the period of cave occupation. Dates from earlier measurements are Z-1641: $290 \pm 100$ (Srdoč et al. 1989: 85), indicating an extended period of cave occupation.

\section{Brezovica series}

Wood fragments from the base of a Roman road at Mala Vas near Brezovica $\left(46^{\circ} 1^{\prime} \mathrm{N}, 14^{\circ} 26^{\prime} \mathrm{E}\right)$, $300 \mathrm{~m}$ asl, Slovenia. Collected 1988 by T. Bregant, Faculty of Arts and Sciences, Ljubljana and submitted by A. Šercelj.

Z-2098. Mala Vas, VS-10/3 1

$780 \pm 80$

Wood fragments (Fagus, Corylus, Populus) from 130-150 cm depth, Quadrant III.

\section{Z-2099. Mala Vas, VS-10/3 2}

$580 \pm 80$

Wood fragment (Betula) from $125 \mathrm{~cm}$ depth, Quadrant III.

Comment (A.Š.): Expected age: Roman period.

Z-2113. Plav

$>37,000$

Fossil wood (Juniperus sp.) from a Pleistocene terrace at Plav $\left(42^{\circ} 35^{\prime} \mathrm{N}, 19^{\circ} 38^{\prime} \mathrm{E}\right)$, Montenegro. Collected 1989 by J. Kunaver, Faculty of Arts and Sciences, Ljubljana and submitted by A. Šercelj.

\section{Z-2114. Bukovnica}

$5650 \pm 110$

Charcoal from a Neolithic site, Quadrant 224, Mkv 64-66, 74-76 at Bukovnica near Dobrovnik $\left(45^{\circ} 39^{\prime} \mathrm{N}, 16^{\circ} 22^{\prime} \mathrm{E}\right), 280-300 \mathrm{~m}$ asl, $15 \mathrm{~km}$ east of Murska Sobota. Collected 1989 by I. Šavelj, Regional Museum, Murska Sobota and submitted by A. Šercelj.

Comment (I.Š.): Expected age: Neolithic. 
Z-2125. Statue of King David's head

$200 \pm 110$

Sample is from a wooden (oak or walnut) statue of King David's head, from a private collection. Collected and submitted 1989 by I. Krtalić, Zagreb.

\section{Zagreb - Grič series}

Charcoal from a fireplace, Clarissa monastery, Grič, Upper Town, Zagreb $\left(45^{\circ} 50^{\prime} \mathrm{N}, 16^{\circ} 0^{\prime} \mathrm{E}\right)$, $150 \mathrm{~m}$ asl, Croatia. Collected and submitted 1989 by N. Majnarić-Pandžić, Department of Archaeology, University of Zagreb.

\section{Z-2130. Zagreb - Grič 1}

$660 \pm 120$

Charcoal from Pit S, 180-183 cm depth.

\section{Z-2129. Zagreb - Grič 2}

$2050 \pm 90$

Charcoal from Fireplace A.

\section{Z-2133. Zagreb - Grič 3}

$2810 \pm 130$

Charcoal from Trench B2/3, $174.8 \mathrm{~cm}$ depth.

Comment (N.M.-P.): Expected age: Middle Ages (15th or 16th century).

Z-2159. Barice

$2990 \pm 90$

Charcoal from a cremation site in the central part of Tumulus I at Barice near Laminci village, Jaružani $\left(45^{\circ} 6^{\prime} 15^{\prime} \mathrm{N}, 17^{\circ} 22^{\prime} \mathrm{E}\right), 92 \mathrm{~m}$ asl, Bosnia. Collected and submitted 1989 by B. Čović, Sarajevo Museum (Đurdjević 1987).

Comment (B.C.): The tumulus is characteristic of the newly discovered Barice - Gredjani cultural group. Expected age: $3300-3500$ BP.

\section{Z-2156. Kistanje}

$350 \pm 100$

Charcoal from the yard of St. Archangels monastery, on the Krka River bank near Kistanje $\left(43^{\circ} 59^{\prime} \mathrm{N}, 16^{\circ} 0^{\prime} \mathrm{E}\right)$, Bukovica, Croatia. Collected and submitted 1989 by B. Uršić, Regional Institute for Protection of Cultural Monuments, Zagreb.

Comment (B.U.): Expected age: AD 9 to 13th century.

\section{Kalnik - Igrišče series}

Charcoal from systematic excavations of a Late Bronze Age settlement on Mt. Kalnik, Igrišče $\left(46^{\circ} 05^{\prime} \mathrm{N}, 16^{\circ} 27^{\prime} \mathrm{E}\right), 400 \mathrm{~m}$ asl, north Croatia. Collected and submitted 1989 by N. MajnarićPandžić.

Charcoal from Hearth 3.

Z-2163. Kalnik - Igrišče A1 A10

$2890 \pm 90$

Charcoal, $0.40 \mathrm{~m}$ depth. 
Z-2161. Kalnik - Igrišče K1 A9

$2980 \pm 70$

Charcoal, $0.59 \mathrm{~m}$ depth.

Z-2162. Kalnik - Igrišče K1 B8

$2650 \pm 60$

Charcoal, $1.20 \mathrm{~m}$ depth

Comment (N.M.-P.): Expected age: 13th to 11th century BC.

\section{Slavonska Požega series}

Wood, bones and charcoal from Sv. Lovro medieval church in Slavonska Požega $\left(45^{\circ} 20^{\prime} \mathrm{N}\right.$, $17^{\circ} 41^{\prime} E$ ), Slavonia, Croatia (Langhamer 1966; Degmedžić 1977; Horvat \& Mirnik 1977). Samples were dated to determine the beginning of church construction. Collected and submitted 1989 by I. Srša, Croatian Institute for Restoration, Zagreb.

\section{Z-2168. Slavonska Požega 1}

$560 \pm 80$

Wooden scaffolding from inside the western gable. An earlier measurement was Z-1921: $790 \pm 100$ (Srdoč et al. 1989: 86-87).

Comment (I.Š.): Expected age: 700 BP.

\section{Z-2169. Slavonska Požega 2}

$700 \pm 90$

Wooden beam, part of western gable.

Comment (I.Š.): Expected age: 700 BP.

Z-2171. Slavonska Požega 3

$400 \pm 90$

Charred wood from the roof or floor, buried in soil at $0.40 \mathrm{~m}$ depth.

Comment (I.Š.): Expected age: 500-550 BP.

\section{Z-2172. Slavonska Požega 4}

Wood fragments of a coffin from Trench 8 , Grave C.

Comment (I.Š.): Expected age: 200-450 BP.

\section{Z-2175. Slavonska Požega 6}

$270 \pm 80$

Human bones in soil from Trench 2, Grave $2 \mathrm{f}$.

Comment (I.Š.): Expected age: maximum 700 BP.

\section{Z-2176. Slavonska Požega 7}

$320 \pm 60$

Human bones from a stone-walled grave, Trench 4.

Comment (I.Š.): Expected age: 500 BP.

\section{Z-2174. Slavonska Požega 8}

$370 \pm 80$

Wooden beam from the northern wall in the aisle. 
Z-2170. Slavonska Požega 9

$690 \pm 80$

Wooden beam from the eastern gable.

Comment (I.Š.): Expected age: 700 BP.

Z-2177. Slavonska Požega 10

$110 \pm 80$

Human bones from Grave C.

\section{Stari grad series}

Fragments of charred wood from an ancient Greek settlement near Stari grad $\left(43^{\circ} 10^{\prime} \mathrm{N}, 16^{\circ} 36^{\prime} \mathrm{E}\right)$, Hvar Island, Croatia. Collected and submitted 1989 by J. Jelčić, Institute for Preservation of Cultural Monuments, Split. Samples were dated to determine the time of destruction of this settlement.

Z-2178. Stari Grad 1

$2540 \pm 70$

Fragments of charred wood from a Hellenistic house yard, $2 \mathrm{~m}$ depth.

Z-2183. Stari Grad 2

$1330 \pm 80$

Fragments of charred wood in front of St. John's Early Christian Church.

Z-2181. Zadar

$800 \pm 80$

Wooden fragments covered with mortar from an arch in a crypt, Sv. Stošija Cathedral, Zadar $\left(45^{\circ} 07^{\prime} \mathrm{N}, 15^{\circ} 15^{\prime} \mathrm{E}\right)$, south Croatia. Collected and submitted 1989 by M. Domijan, Institute for Preservation of Cultural Monuments, Zadar.

Comment (M.D.): Expected age: Middle Ages.

\section{Nova Rača series}

Human bones from Nova Rača church near Bjelovar $\left(45^{\circ} 47^{\prime} \mathrm{N}, 16^{\circ} 56^{\prime} \mathrm{E}\right), 175 \mathrm{~m}$ asl, central Croatia. Collected and submitted 1989 by G. Jakovljević, Bjelovar Museum. Samples were dated to determine the chronology of the strata containing human bones.

\section{Z-2184. Nova Rača}

$380 \pm 60$

Bones buried in clay, $1.81 \mathrm{~m}$ below the church sacristy.

Z-2187. Nova Rača

$610 \pm 60$

Bones at $1.20 \mathrm{~m}$ below ground level from a necropolis outside the church, near the south wall.

Z-2255. Nova Rača

$80 \pm 80$

Bones from Grave 1 in the churchyard, $110 \mathrm{~cm}$ depth.

\section{Z-2256. Nova Rača}

$70 \pm 80$

Bones from Grave 2 in the churchyard, $87 \mathrm{~cm}$ depth. 
Z-2258. Nova Rača

$470 \pm 70$

Bones from plundered Grave 2, below the church sacristy, $100 \mathrm{~cm}$ depth.

Z-2260. Nova Rača

$570 \pm 80$

Bones from plundered Grave 18 , below the church sacristy, $30 \mathrm{~cm}$ depth.

Comments: (G.J.): Expected age: Middle Ages. (G.J.): Date confirms results of archaeological and historical investigations.

\section{Grabovac series}

Samples of charcoal from Eneolithic pits, from Grabovac brickyard near Đakovo $\left(45^{\circ} 18^{\prime} \mathrm{N}\right.$, $18^{\circ} 27^{\prime} \mathrm{E}$ ). Collected 1990 by I. Pavlović, Regional Museum Đakovo, and submitted by S. Forenbacher, Department of Archaeology, University of Zagreb.

Z-2239.

$4760 \pm 150$

Charcoal from Pit 4.

Z-2240.

Charcoal from Pit 3B.

Comment (I.P.): Expected age: coeval with the Baden culture (Durman \& Obelić 1989).

\section{Vinkovci series}

Charcoal from the floor of a primitive Vučedol-type house near Vinkovci $\left(45^{\circ} 16^{\prime} \mathrm{N}, 18^{\circ} 49^{\prime} \mathrm{E}\right)$, $\approx 20 \mathrm{~km}$ west of Eneolithic site, Vučedol, east Croatia. Collected 1977 and submitted 1990 by A. Durman, Department of Archaeology, University of Zagreb. Samples were dated to determine the duration of the Vučedol culture in this area. Dates correspond to earlier measurements, Z-1817, -1818 (Srdoč et al. 1989: 87).

\section{Z-2189. Vinkovci, Sample A}

$3940 \pm 90$

Charcoal.

\section{Z-2190. Vinkovci, Sample B}

$4080 \pm 130$

Charcoal.

\section{Z-2238. Krapina}

$1320 \pm 140$

Fragments of fossil oak from the Krapina River bed near Zaprešić $\left(45^{\circ} 48^{\prime} \mathrm{N}, 15^{\circ} 58^{\prime} \mathrm{E}\right)$, Croatia. Collected 1989 and submitted 1990 by D. Stošić.

\section{Z-1926. Koprivnički bregi}

$4470 \pm 140$

Charcoal mixed with soil from Pit DE/87 at Seče near Koprivnički bregi $\left(46^{\circ} 10^{\prime} \mathrm{N}, 14^{\circ} 34^{\prime} \mathrm{E}\right)$, Koprivnica, Croatia. Collected and submitted 1987 by Z. Marković, Koprivnica Town Museum.

Comment (Z.M.): Expected age: 3000-4000 BC. 


\section{HUNGARY}

\section{Gorzsa series}

Charcoal from tell-type Late Neolithic settlements from the Tisza region, southeast Hungary (Horvath, 1982, 1986, 1987; Benkö et al. 1989). Collected by F. Horvath, Mora Ferenc Museum, Szeged, and submitted 1987 by L. Benkö, Institute of Isotopes, Hungarian Academy of Sciences, Budapest.

\section{Z-2007. Tápé - Lebö 1}

$5870 \pm 110$

Charcoal from Block I, Layer 2-3, Quadrant 4/A at Tápé - Lebö $\left(46^{\circ} 16^{\prime} \mathrm{N}, 20^{\circ} 17^{\prime} \mathrm{E}\right), 82 \mathrm{~m}$ asl.

Z-2008. Szegvar - Tuszkoves 2

$6550 \pm 160$

Charcoal from Block I, Layer 29, Quadrant 105 at Szegvar - Tuszkoves (46 $36^{\prime} 53^{\prime \prime} \mathrm{N}$, $\left.20^{\circ} 15^{\prime} 42^{\prime \prime} \mathrm{E}\right), 87 \mathrm{~m}$ asl.

\section{Z-2009. Gorzsa 3}

$5610 \pm 110$

Charcoal from Block XIV, Layer 6-7, Phase 6 at Gorzsa $\left(46^{\circ} 16^{\prime} 25^{\prime \prime} \mathrm{N}, 20^{\circ} 17^{\prime} 26^{\prime \prime} \mathrm{E}\right), 81 \mathrm{~m}$ asl.

\section{Z-2010. Gorzsa 4}

$5820 \pm 110$

Charcoal from Block VI, House 2, Phase C at Gorzsa.

\section{Z-2011. Gorzsa 5}

Charcoal from Block XVIII, Layers 24-25, Phase D at Gorzsa.

Comment (D.S.): Dates are discussed in Benkö et al. (1989).

\section{HYDROGEOLOGICAL AND GROUNDWATER SAMPLES}

YUGOSLAVIA

\section{Ljubljansko barje series}

Groundwater from Ljubljansko barje peat bog $\left(45^{\circ} 58^{\prime} \mathrm{N}, 14^{\circ} 32^{\prime} \mathrm{E}\right)$, Slovenia. Groundwater issues from a confined limestone aquifer. The total dissolved inorganic carbon (TDIC) consists predominantly of Ca-bicarbonate. Collected by J. Prestor and submitted 1988 by M. Veselič, Geological Institute, Ljubljana, Slovenia. This groundwater was studied in relation to a potable waterworks.

\section{Z-2027. P-2}

$21.1 \pm 1.3 \mathrm{pMC}$

Groundwater. Tritium activity: $<0.2 \mathrm{~Bq} /$ liter.

Z-2028. Ž-4

$76.1 \pm 1.4 \mathrm{pMC}$

Groundwater. Tritium activity: $3.3 \pm 0.2 \mathrm{~Bq} / \mathrm{liter}$.

Z-2029. V-3

Groundwater. Tritilim activity: $<0.2 \mathrm{~Bq} /$ liter. 


\section{Bačka series}

Samples were part of a hydrogeological and hydrodynamic study of groundwater from subartesian water-bearing horizons with intergranular porosity Bačka, Vojvodina. Collected and submitted 1988 by M. Lazarević, "Jaroslav Černi” Institute, Belgrade.

\section{Z-2030. Savino Selo 1}

$74.2 \pm 1.1 \mathrm{pMC}$

Groundwater from a phreatic well, $206.4 \mathrm{~m}$ deep, at Savino Selo $\left(45^{\circ} 30^{\prime} \mathrm{N}, 19^{\circ} 33^{\prime} \mathrm{E}\right)$, $36.06-61.35 \mathrm{~m}$ depth $\left(\mathrm{pH}=7.5, \mathrm{HCO}_{3}=481 \mathrm{mg} / \mathrm{liter}\right)$.

\section{Z-2031. Titov Vrbas 2}

Spring water supplying Titov Vrbas waterworks $\left(45^{\circ} 34^{\prime} \mathrm{N}, 19^{\circ} 37^{\prime} \mathrm{E}\right), 121.60-144.70 \mathrm{~m}$ depth. Subartesian aquifer $156 \mathrm{~m}$ deep $\left(\mathrm{pH}=7.7, \mathrm{HCO}_{3}=518 \mathrm{mg} / \mathrm{liter}\right)$.

\section{Z-2032. Kumra 3}

$4.4 \pm 0.8 \mathrm{pMC}$

Groundwater from a subartesian well, $220 \mathrm{~m}$ deep, at Kumra, $185.00-198.50 \mathrm{~m}$ depth.

\section{Z-2033. Zmajevo 4}

$2.8 \pm 0.8 \mathrm{pMC}$

Artesian mineral water from a well, $369.80 \mathrm{~m}$ deep, at Zmajevo $\left(45^{\circ} 27^{\prime} \mathrm{N}, 19^{\circ} 42^{\prime} \mathrm{E}\right)$, 348.90-367.90 m depth.

\section{Z-2034. Bački Jarak 5}

$1.9 \pm 0.8 \mathrm{pMC}$

Water from a subartesian well at Bački Jarak $\left(45^{\circ} 22^{\prime} \mathrm{N}, 19^{\circ} 53^{\prime} \mathrm{E}\right), 158.16-181.50 \mathrm{~m}$ depth.

\section{Z-2035. Bački Jarak 6}

$6.4 \pm 0.8 \mathrm{pMC}$

Water from a subartesian well at Bački Jarak, $130 \mathrm{~m}$ deep, 90-123 m depth.

\section{Surdulica series}

Water, peat, soil and plants from Surdulica, one of the largest geothermal systems in southeast Yugoslavia, Mt. Besna kobila massif, $1922 \mathrm{~m}$ asl, part of the geotectonic region of Serbia and Macedonia (Milovanović et al. 1989). The hydrogeothermal system is in the neogenfissured granodiorite aquifer, surrounded by crystalline schists. The system produces cold water $(\approx 100$ liter/s, alkalinity $61-140 \mathrm{mg} /$ liter $)$ and geothermal water $(\sim 70$ liter/s, alkalinity $260-267 \mathrm{mg} /$ liter). This series is part of an investigation of the mechanism of water formation in the Surdulica geothermal system. Collected and submitted 1988 by M. Hadžišehović, Institute Boris Kidrič, Belgrade.

\section{Z-2066. Kula BK-6}

$38.8 \pm 1.3 \mathrm{pMC}$

Water from a borehole, $112 \mathrm{~m}$ deep, at Kula near Kriva Feja $\left(42^{\circ} 34^{\prime} \mathrm{N}, 22^{\circ} 09^{\prime} \mathrm{E}\right), 1354 \mathrm{~m}$ asl, Mt. Besna kobila.

\section{Z-2073. Bujanovac BH-7 1}

$$
3.3 \pm 0.8 \mathrm{pMC}
$$

Geothermal water $\left(43^{\circ} \mathrm{C}, \mathrm{pH}=6-6.5\right)$ from Borehole BH-7, $556 \mathrm{~m}$ deep, at Bujanovac $\left(42^{\circ} 25^{\prime} \mathrm{N}\right.$, $\left.21^{\circ} 45^{\prime} \mathrm{E}\right), 410 \mathrm{~m}$ asl, south Serbia. Collected 12-15 July 1988. 


\section{Z-2137. Bujanovac BH-7 2}

Groundwater from Borehole BH-7. Collected 20-25 May 1989.

\section{Z-2074. Valjavica 22}

$67.0 \pm 1.1 \mathrm{pMC}$

Water from the Valjavica well $\left(13^{\circ} \mathrm{C}, \mathrm{pH}=5.5\right)$ near Surdulica $\left(42^{\circ} 40^{\prime} \mathrm{N}, 22^{\circ} 10^{\prime} \mathrm{E}\right), 910 \mathrm{~m}$ asl, south Serbia.

\section{Z-2135. Tolovački potok}

$61.3 \pm 1.0 \mathrm{pMC}$

Water from the Tolovac catchment near Vranjska Banja spa $\left(42^{\circ} 30^{\prime} \mathrm{N}, 21^{\circ} 48^{\prime} \mathrm{E}\right), 577 \mathrm{~m}$ asl, Mt. Besna kobila.

Z-2136. Vranjska Banja V6-3

$$
4.8 \pm 0.8 \mathrm{pMC}
$$

Geothermal water $\left(67^{\circ} \mathrm{C}\right)$ from a borehole, $450 \mathrm{~m}$ deep, at Vranjska Banja spa $\left(42^{\circ} 30^{\prime} \mathrm{N}\right.$, $\left.21^{\circ} 48^{\prime} \mathrm{E}\right), 406 \mathrm{~m}$ asl.

\section{Z-2134. Ribarska Banja RB-5}

$11.1 \pm 0.9 \mathrm{pMC}$

Geothermal water $\left(40^{\circ}-52^{\circ} \mathrm{C}\right)$ from Ribarska Banja spa $\left(43^{\circ} 24^{\prime} \mathrm{N}, 21^{\circ} 31^{\prime} \mathrm{E}\right), 430 \mathrm{~m}$ asl, Mt. Jastrebac foothills.

\section{Z-2138. Toplac}

Geothermal water $\left(20.5^{\circ}-24^{\circ} \mathrm{C}\right)$ from Toplac $\left(42^{\circ} 33^{\prime} \mathrm{N}, 21^{\circ} 57^{\prime} \mathrm{E}\right), 393 \mathrm{~m}$ asl.

\section{Z-2067. Ladne Vode ML-1}

$2.6 \pm 0.8 \mathrm{pMC}$

Water from a borehole, Mlava River valley near Gornjačka klisura $\left(42^{\circ} 45^{\prime} \mathrm{N}, 20^{\circ} 25^{\prime} \mathrm{E}\right)$, southeast Serbia.

\section{Z-2071. Ždrelo 11}

$104.1 \pm 1.2 \mathrm{pMC}$

Water from a spring $(\mathrm{pH}=6)$ at Ždrelo village near Petrovac $\left(44^{\circ} 18^{\prime} \mathrm{N}, 21^{\circ} 32^{\prime} \mathrm{E}\right), 710 \mathrm{~m}$ asl, on the Mlava River.

\section{Z-2072. Stari Glog 1}

$94.1 \pm 1.5 \mathrm{pMC}$

Water $\left(17.6^{\circ} \mathrm{C}, \mathrm{pH}=5.5-6\right)$ from a catchment at Stari Glog $\left(42^{\circ} 28^{\prime} \mathrm{N}, 22^{\circ} 08^{\prime} \mathrm{E}\right), 710 \mathrm{~m}$ asl, Mt. Besna kobila.

\section{Velenje series}

Groundwater from several boreholes in brown coal (lignite) at Velenje coal mine $\left(46^{\circ} 21^{\prime} \mathrm{N}\right.$, $15^{\circ} 07^{\prime} \mathrm{E}$ ), Slovenia. Collected and submitted 1988 by M. Veselič, Geological Institute Ljubljana. This was part of an investigation of the origin of groundwater flooding the coal mine.

\section{Z-2075. VAS-3}

Groundwater from Borehole VAS-3. Tritium activity: $<0.2 \mathrm{~Bq} /$ liter.

$$
\begin{aligned}
2.2 & \pm 0.9 \mathrm{pMC} \\
\delta^{13} \mathrm{C} & =-5.2 \% 0
\end{aligned}
$$




\section{Z-2076. VAS-4}

Groundwater from Borehole VAS-4. Tritium activity: $0.6 \pm 0.2 \mathrm{~Bq} / \mathrm{liter}$.

Z-2077. VAS-5

Groundwater from Borehole VAS-5. Tritium activity: $0.6 \pm 0.2 \mathrm{~Bq} /$ liter.

\section{Z-2078. VL-16}

Groundwater from Borehole VAS-16. Tritium activity: $<0.2$ Bq/liter.

Z-2079. VAS-6

Groundwater from Borehole VAS- 6 . Tritium activity: $0.5 \pm 0.2 \mathrm{~Bq} /$ liter.

Z-2080. VAS-7

Groundwater from Borehole VAS-7. Tritium activity: $<0.2 \mathrm{~Bq} / \mathrm{liter}$.

Z-2081. VL-15

Groundwater from Borehole VL-15. Tritium activity: $<0.2 \mathrm{~Bq} /$ liter.

\section{Z-2082. VL-18}

Groundwater from Borehole VL-18. Tritium activity: $0.8 \pm 0.2 \mathrm{~Bq} /$ liter.

\section{Z-2083. VS-17}

Groundwater from Borehole VS-17. Tritium activity: $<0.2 \mathrm{~Bq} /$ liter.

Z-2084. VS-19

Groundwater from Borehole VS-19. Tritium activity: $<0.2 \mathrm{~Bq} /$ liter.

Z-2085. VA-21

Groundwater from Borehole VA-21. Tritium activity: $2.2 \pm 0.2 \mathrm{~Bq} /$ liter.

Z-2086. VS-20

Groundwater from Borehole VS-20. Tritium activity: $<0.2 \mathrm{~Bq} /$ liter.

Z-2087. VA-22

Groundwater from Borehole VA-22. Tritium activity: $1.0 \pm 0.2 \mathrm{~Bq} /$ liter.

\section{Z-2088. VS-23}

Groundwater. Tritium activity: $<0.2 \mathrm{~Bq} /$ liter.
$15.3 \pm 1.4 \mathrm{pMC}$

$\delta^{13} \mathrm{C}=-7.9 \%$

$13.1 \pm 1.1 \mathrm{pMC}$

$\delta^{13} \mathrm{C}=-8.6 \%$

$0.0 \pm 0.8 \mathrm{pMC}$

$\delta^{13} C=0.6 \%$

$13.9 \pm 1.0 \mathrm{pMC}$

$\delta^{13} \mathrm{C}=-8.6 \%$

$5.9 \pm 1.3 \mathrm{pMC}$

$\delta^{13} \mathrm{C}=-8.9 \%$

$2.2 \pm 0.8 \mathrm{pMC}$

$26.0 \pm 1.0 \mathrm{pMC}$

$\delta^{13} \mathrm{C}=-11.2 \%$

$8.0 \pm 0.8 \mathrm{pMC}$

$\delta^{13} C=-19.1 \%$ 。

$6.7 \pm 0.8 \mathrm{pMC}$

$\delta^{13} \mathrm{C}=-15.9 \%$ o

$42.5 \pm 1.1 \mathrm{pMC}$

$\delta^{13} C=-11.7 \%$

$5.6 \pm 0.8 \mathrm{pMC}$

$\delta^{13} \mathrm{C}=-18.2 \%$

$30.3 \pm 0.9 \mathrm{pMC}$

$\delta^{13} \mathrm{C}=-11.8 \%$

$30.8 \pm 1.2 \mathrm{pMC}$

$\delta^{13} C=-13.6 \%$

General Comment: Tritium activity in waters with relatively low ${ }^{14} \mathrm{C}$ activity indicates mixing of old and recent water, and the age of these waters cannot be determined on the basis of their ${ }^{14} \mathrm{C}$ content. 


\section{Niška Banja series}

Thermomineral waters from Niška Banja spa $\left(43^{\circ} 18^{\prime} \mathrm{N}, 22^{\circ} 01^{\prime} \mathrm{E}\right)$ near Niš, Serbia. Collected and submitted 1988 by M. Milivojević, Faculty of Mineralogy, Geology and Petrology Engineering, University of Belgrade. This was a hydrogeological investigation of the origin of thermomineral water in a low-temperature system of Niška Banja spa.

\section{Z-2101. Glavno vrelo}

$49.7 \pm 1.2 \mathrm{pMC}$

Water from Glavno vrelo (main spring). Tritium activity: $0.7 \pm 0.1 \mathrm{~Bq} /$ liter.

\section{Z-2102. Suva Banja}

$51.2 \pm 1.3 \mathrm{pMC}$

Water from Suva Banja spa. Tritium activity: $0.7 \pm 0.2 \mathrm{~Bq} / \mathrm{liter}$.

\section{Z-2103. IEBNB-1}

$14.6 \pm 1.1 \mathrm{pMC}$

Water from borehole IEBNB-1, $520 \mathrm{~m}$ depth, $210 \mathrm{~m}$ asl.

Z-2104. GC-3

$3.0 \pm 0.8 \mathrm{pMC}$

Mineral water from a 370-m-deep borehole, $150 \mathrm{~m}$ depth, $250 \mathrm{~m}$ asl, at Gornje Crniljevo near Osečina $\left(19^{\circ} 40^{\prime} \mathrm{N}, 44^{\circ} 29^{\prime} \mathrm{E}\right)$, west Serbia. Water was dated in a hydrogeological investigation of the origin of the mineral water and the possibility of exploration. Tritium activity: $<0.2 \mathrm{~Bq} /$ liter.

\section{CZECHOSLOVAKIA}

\section{Czechoslovakia series}

Groundwater samples from central Bohemian Karst and Mt. Male Karpaty, west Slovakia. Collected and submitted 1987 by N. Horvatinčić and J. Šilar, Faculty of Science, Charles University, Praha, Czechoslovakia. Dating of recent tufa helps determine the initial ${ }^{14} \mathrm{C}$ activity of groundwater.

\section{Z-1972. Cisarska Rokle}

$78.7 \pm 1.2 \mathrm{pMC}$

Water from a spring at Cisarska Rokle, near Srbsko $\left(49^{\circ} 52^{\prime} \mathrm{N}, 17^{\circ} 02^{\prime} \mathrm{E}\right), 310 \mathrm{~m}$ asl. Tritium activity: $4.7 \mathrm{~Bq} /$ liter.

\section{Z-1973. Koda}

$87.1 \pm 1.1 \mathrm{pMC}$

Water from a catchment at Koda, near Srbsko $\left(49^{\circ} 52^{\prime} \mathrm{N}, 17^{\circ} 02^{\prime} \mathrm{E}\right), 320 \mathrm{~m}$ asl. Tritium activity: 5.7 Bq/liter.

\section{Z-1974. Babina}

$76.4 \pm 1.1 \mathrm{pMC}$

Water from a catchment at Babina, near Hradište pod Vratnom, Mt. Male Karpaty $\left(48^{\circ} 33^{\prime} \mathrm{N}\right.$, $\left.17^{\circ} 30^{\prime} \mathrm{E}\right)$. Tritium activity: $1.7 \mathrm{~Bq} /$ liter.

\section{Z-1975. Sv. Jan pod Skalou}

$82.8 \pm 1.1 \mathrm{pMC}$

Water from a spring at Sv. Jan pod Skalou $\left(49^{\circ} 53^{\prime} \mathrm{N}, 17^{\circ} 02^{\prime} \mathrm{E}\right)$. Tritium activity: $5.5 \mathrm{~Bq} / \mathrm{liter}$. 


\section{GEOLOGICAL SAMPLES}

\section{YUGOSLAVIA}

\section{Bačka series}

Fossil soil was collected from boreholes in Bačka, Vojvodina for geotectonic investigations in northeast Serbia. Collected and submitted Sep. 1987 by M. Galečić, Geological Institute Belgrade.

\section{Z-1952. Mali Beograd}

$$
33,800 \underset{-2300}{+3200}
$$

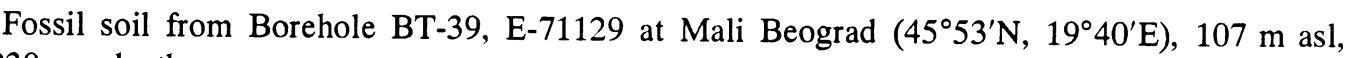
$1030 \mathrm{~cm}$ depth.

\section{Z-1953. Dušanovo}

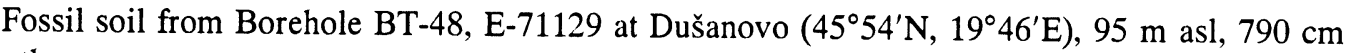
depth.

\section{Z-1954. Gunaroš}

Fossil soil from Borehole BT-53, E-71153 at Gunaroš (45 $\left.46^{\prime} \mathrm{N}, 19^{\circ} 50^{\prime} \mathrm{N}\right), 100 \mathrm{~m}$ asl, $960 \mathrm{~cm}$ depth.

\section{Z-1955. Mileševo}

$29,900 \pm 2600$

Fossil soil from Borehole BT-54, E-71167 at Mileševo (45 $\left.44^{\prime} \mathrm{N}, 19^{\circ} 48^{\prime} \mathrm{E}\right), 104 \mathrm{~m}$ asl, $890 \mathrm{~cm}$ depth.

\section{Z-1956. Mileševo}

Fossil soil from Borehole BT-55, E-71162 at Mileševo (97 m asl), $915 \mathrm{~cm}$ depth.

\section{Serbia series}

Clay and peat were collected from Serbia in an investigation of the Quaternary of Serbia, and dated to draft a geological map of Yugoslavia. Collected and submitted 1987 by M. Rakić, Geozavod, Belgrade.

\section{Z-1988. Radinac 079-4-2 4}

Modern

Gray clay, $0.4 \mathrm{~m}$ below moor vegetation from an old bed of the Velika Morava River at Radinac, near Smederevo $\left(44^{\circ} 39^{\prime} \mathrm{N}, 20^{\circ} 55^{\prime} \mathrm{E}\right), 72 \mathrm{~m}$ asl.

\section{Z-1989. Zvečka 078-3-4 17}

$7250 \pm 170$

Organogenic pond clay with fauna from an old meander of the Sava River at Zvečka village near Obrenovac $\left(44^{\circ} 38^{\prime} \mathrm{N}, 20^{\circ} 10^{\prime} \mathrm{E}\right), 73 \mathrm{~m}$ asl, at depth $1.0-1.5 \mathrm{~m}$ below ground, covered by reeds and cultivated land.

Comment: Expected age: 4000-6000. 
Z-1990. Zabrežje 078-3-2 35

$95.3 \pm 1.2 \mathrm{pMC}$

Aleurite with humus at Zabrežje village near Obrenovac $\left(44^{\circ} 41^{\prime} \mathrm{N}, 20^{\circ} 05^{\prime} \mathrm{E}\right), 72 \mathrm{~m}$ asl, at depth $2.0 \mathrm{~m}$, below cultivated land.

Comment: $680 \pm 110$.

Z-1991. Gaj 080-1-4 69

$11,220 \pm 250$

Peat from an old meander of the Danube River at Gaj village, near Kovin $\left(44^{\circ} 48^{\prime} \mathrm{N}, 21^{\circ} 02^{\prime} \mathrm{E}\right)$, $67 \mathrm{~m}$ asl, Banat, Vojvodina, at depth $1.0-1.5 \mathrm{~m}$, below cultivated land.

Comment (M.R.): Expected age: 4000-6000.

\section{Z-1992. Dubovac 080-1-4 70}

$2750 \pm 130$

Sandy peat from an old meander of the Danube River at Dubovac village $\left(44^{\circ} 48^{\prime} \mathrm{N}, 21^{\circ} 10^{\prime} \mathrm{E}\right)$, $69 \mathrm{~m}$ asl, Banat, Vojvodina, from the surface.

Comment (M.R.): Expected age: 4000-6000.

\section{Lake Bled series}

Lake Bled $\left(46^{\circ} 21^{\prime} \mathrm{N}, 14^{\circ} 08^{\prime} \mathrm{E}\right), 500 \mathrm{~m}$ asl. Collected and submitted 1988 by J. Pezdič, Institute Jožef Štefan, Ljubljana, as part of a sedimentological investigation of the origin of the lake marl. The lake marl consists of calcite without detrital minerals. $\delta^{13} \mathrm{C}$ ranges between -1.03 and $-2.36 \%$ vs. PDB.

Z-1993. B-K-2

Lake sediment, $40 \mathrm{~cm}$ depth.

\section{Z-1994. B-83}

Shells from the base of the lake, $2 \mathrm{~m}$ depth.

Z-1995. B-11/2

$23.6 \pm 0.9 \mathrm{pMC}$

Lake sediment, $0-10 \mathrm{~cm}$ depth.

Z-1996. B-11/2

$19.6 \pm 0.8 \mathrm{pMC}$

Lake sediment, $20-30 \mathrm{~cm}$ depth.

Z-1997. B-11/2

$26.2 \pm 0.9 \mathrm{pMC}$

Lake sediment, 50-60 cm depth.

Z-1998. B-11/5

$37.3 \pm 0.9 \mathrm{pMC}$

Lake sediment, $20-30 \mathrm{~cm}$ depth.

Z-1999. B-I-28

$17.3 \pm 0.9 \mathrm{pMC}$

Lake sediment, 5-20 cm depth. 


\section{Z-2001. B-I-28}

$14.7 \pm 0.8 \mathrm{pMC}$

Lake sediment, $20-50 \mathrm{~cm}$ depth.

Z-1969. Selca

$8820 \pm 210$

Clay rich in organic material (humus), from a borehole core at $150 \mathrm{~cm}$ depth, from Selca, near Škofja Loka $\left(45^{\circ} 13^{\prime} 10^{\prime \prime} \mathrm{N}, 14^{\circ} 13^{\prime} 25^{\prime \prime} \mathrm{E}\right), 430 \mathrm{~m}$ asl, Slovenia. Collected and submitted 1988 by A. Šercelj. Sample was dated for a vegetation profile.

Comment (A.Š.): Expected period: Holocene.

\section{Kamniške Alpe series}

Samples were dated in a lithostratigraphic investigation of alpine karst. Collected 1987 by J. Urbanc and submitted 1988 by J. Pezdič.

\section{Z-2002. Kamniška jama cave}

$220 \pm 80$

Humus from Kamniška jama cave $\left(46^{\circ} 21^{\prime} \mathrm{N}, 14^{\circ} 31^{\prime} \mathrm{E}\right), 1100 \mathrm{~m}$ asl.

\section{Z-2003. Ogrlice 2}

Topsoil at Ogrlice $\left(46^{\circ} 20^{\prime} \mathrm{N}, 14^{\circ} 40^{\prime} \mathrm{E}\right), 0-15 \mathrm{~cm}$ depth.

\section{Z-2004. Bela 3}

Topsoil at Bela $\left(46^{\circ} 20^{\prime} \mathrm{N}, 14^{\circ} 37^{\prime} \mathrm{E}\right), 0-15 \mathrm{~cm}$ depth.
Modern $105.2 \pm 1.2 \mathrm{pMC}$

Z-2005. Ljubljansko barje

$2800 \pm 130$

Fragments of wood from Ljubljansko barje peat bog $\left(45^{\circ} 59^{\prime} \mathrm{N}, 14^{\circ} 32^{\prime} \mathrm{E}\right)$. Collected by J. Urbanc and submitted 1988 by J. Pezdič, as part of a palynological investigation of Ljubljansko barje peat bog.

\section{Surdulica series}

This investigation was of ${ }^{14} \mathrm{C}$ activity of peat, soil and plants in the recharge area of the Surdulica aquifer. Collected and submitted 1988 by M. Hadžišehović.

\section{Z-2068. Lake Vlasina}

Modern $111.4 \pm 1.5 \mathrm{pMC}$

Peat from Lake Vlasina shore $\left(42^{\circ} 42^{\prime} \mathrm{N}, 22^{\circ} 20^{\prime} \mathrm{E}\right), 1300 \mathrm{~m}$ asl, southeast Serbia.

Comment (M.H.): Expected age: Holocene.

\section{Z-2069. Lake Vlasina}

Modern $126.1 \pm 1.4 \mathrm{pMC}$

Soil mixed with grass from a clearing near Lake Vlasina $\left(42^{\circ} 42^{\prime} \mathrm{N}, 22^{\circ} 20^{\prime} \mathrm{E}\right) 1295-1300 \mathrm{~m}$ asl, Mt. Besna kobila. 


\section{Z-2070. Lake Vlasina}

Modern

$123.9 \pm 1.5 \mathrm{pMC}$

Various unidentified plants from the Lake Vlasina area.

Comment: Result is close to the atmospheric ${ }^{14} \mathrm{C}$ activity.

Z-2089. Unije Island 51-4-J

$5700 \pm 150$

Loess concretions (loess kindchen) from Unije Island $\left(44^{\circ} 35^{\prime} \mathrm{N}, 14^{\circ} 18^{\prime} \mathrm{E}\right), 9 \mathrm{~m}$ asl, Adriatic Sea, Croatia. Collected for a stratigraphic and sedimentologic investigation. Collected and submitted 1988 by Z. Velimirović, INA-Project, Zagreb.

\section{Z-2097. Sesalac cave}

$64.4 \pm 1.1 \mathrm{pMC}$

Speleothem deposited on a wall of the main shaft of a short tunnel, Sesalac cave near Soko Banja $\left(43^{\circ} 41^{\prime} 53^{\prime \prime} \mathrm{N}, 21^{\circ} 59^{\prime} 26^{\prime \prime} \mathrm{E}\right), 602 \mathrm{~m}$ asl, Serbia. Sample was dated to determine the time of gravel filling (Petrović 1984). Collected and submitted 1987 by D. Gavrilović, Faculty of Natural Sciences and Mathematics, Belgrade.

Comments (D.G.): Expected age: Pleistocene. (D.S.): assuming $\mathrm{A}_{\mathrm{o}}=100 \mathrm{pMC}$, the speleothem ${ }^{14} \mathrm{C}$ age is $3500 \pm 140 \mathrm{BP}$.

\section{Rijeka series}

Organic fraction from Boreholes A-2 and S-2 at Rijeka $\left(45^{\circ} 18^{\prime} \mathrm{N}, 14^{\circ} 25^{\prime} \mathrm{E}\right)$, southwest Croatia. Collected and submitted 1987 and 1989 by E. Pavlovec, Institute for Civil Engineering, Rijeka. Collected for geotechnical investigations of the city development area.

\section{Z-2036. Rijeka}

$6410 \pm 160$

Organic mud from Borehole A-2, $8 \mathrm{~m}$ depth.

\section{Z-2105. 1}

$3680 \pm 140$

Organic fraction of soil, $25 \mathrm{~m}$ depth.

\section{Z-2106. 2}

$1330 \pm 120$

Organic fraction of soil, $5.7 \mathrm{~m}$ depth.

Comment: Expected age: Holocene.

\section{Lake Kozjak series}

Samples of calcareous lake sediment from the Plitvice Lakes $\left(44^{\circ} 50^{\prime} \mathrm{N}, 15^{\circ} 35^{\prime} \mathrm{E}\right)$ central Croatia were collected with a hand corer February 1989 by scuba diver, D. Petricioli, Center for Marine Research, Rudjer Bošković Institute, Zagreb.

TABLE 1. Lake Kozjak Sediment Core

\begin{tabular}{ccclc}
\hline Sample no. & Depth $(\mathrm{cm})$ & pMC & Age (BP) & $\delta^{13}$ C\%o (PDB) \\
\hline Z-2116 & $5-10$ & $79.9 \pm 0.6$ & Modern & -8.6 \\
Z-2117 & $10-15$ & $74.0 \pm 0.6$ & $214 \pm 70$ & -8.9 \\
Z-2118 & $15-20$ & $74.4 \pm 0.6$ & $170 \pm 70$ & -9.1 \\
Z-2119 & $20-25$ & $73.6 \pm 0.8$ & $260 \pm 90$ & -9.2 \\
Z-2120 & $25-29$ & $73.3 \pm 0.8$ & $290 \pm 90$ & -9.1 \\
\hline
\end{tabular}


Z-2127. Lake Kozjak

$82.1 \pm 1.0 \mathrm{pMC}$

The organic part of the sediment was used to date the organic residue after chemical pretreatment of samples Z-2117, -2119 and -2120 .

Comment: Initial ${ }^{14} \mathrm{C}$ activity, $\mathrm{A}_{0}=76 \%$, was determined by two independent methods (Srdoč et al. 1986b; Krajcar Bronić et al. 1992). Table 1 shows the results.

\section{Plitvice travertines series}

Travertine from the Plitvice Lakes $\left(44^{\circ} 50^{\prime} \mathrm{N}, 15^{\circ} 35^{\prime} \mathrm{E}\right)$ central Croatia. Collected and submitted 1989 by H. Chafetz, Department of Geosciences, University of Houston, D. Srdoč and N. Horvatinčić.

\section{Z-2142. Plitvički Ljeskovac}

$0.0 \pm 0.5 \mathrm{pMC}$

Compact crystalline flowstone from old travertine barrier near Plitvički Ljeskovac.

\section{Z-2143. Smolčića cave}

$1.7 \pm 0.5 \mathrm{pMC}$

Compact, hard, partly porous travertine above Smolčića cave.

\section{Z-2144. Bijela rijeka}

$1.4 \pm 0.6 \mathrm{pMC}$

Crystalline flowstone from an inactive travertine barrier above Bijela rijeka brook.

General Comment (D.S.): Samples were collected for U/Th analysis. The ${ }^{14} \mathrm{C}$ content indicates the degree of contamination with recent carbonates.

\section{Z-2124. Rovinj}

$9270 \pm 250$

Peat from a borehole in marine sediment, $98-108 \mathrm{~cm}$ depth, at station 109 in the North Adriatic Sea near Rovinj $\left(45^{\circ} 05^{\prime} \mathrm{N}, 13^{\circ} 37^{\prime} \mathrm{E}\right)$. Collected and submitted 1989 by S. Puškarić, Center for Marine Research, Rudjer Bošković Institute, Zagreb.

\section{Krka River series}

Tufa from Krka River near Skradin $\left(43^{\circ} 49^{\prime} \mathrm{N}, 15^{\circ} 55^{\prime} \mathrm{E}\right)$, Croatia. Collected and submitted 1989 by D. Srdoč, N. Horvatinčić, S. Golubić, Boston University and A. Plenković, University of Zagreb, and 1991 by D. Srdoč and T. Bjedov, Elektroprojekt, Zagreb.

Z-2253. 1

Recent tufa near Roški slap waterfall.

Z-2254. 2

Recent tufa near Roški slap waterfall, downstream from Z-2253.

Z-2264. 3

Recent tufa, Lake Mlinarsko.

Z-2141. 4

Recent tufa from a barrier, surface layer, downstream from Lake Mlinarsko.
$76.1 \pm 0.8 \mathrm{pMC}$

$79.7 \pm 0.8 \mathrm{pMC}$

$81.7 \pm 0.8 \mathrm{pMC}$

$83.1 \pm 0.8 \mathrm{pMC}$ 
Recent tufa, Skradinski buk waterfall.

Recent tufa, Skradinski buk waterfall.

\section{Z-2140. 7}

$95.9 \pm 0.8 \mathrm{pMC}$

Recent tufa, Skradinski buk waterfall.

Comment (D.S.): Measurements were of recent tufa activity in the Krka River, north Dalmatia, Croatia. ${ }^{14} \mathrm{C}$ activity of dissolved inorganic carbon (DIC) increases downstream due to the exchange with more active atmospheric $\mathrm{CO}_{2}$ (Srdoč et al. 1986a). Recent tufa precipitated from DIC reflects the DIC ${ }^{14} \mathrm{C}$ activity. We observed an increase of $10 \%$ in ${ }^{14} \mathrm{C}$ activity over a $12-\mathrm{km}$-long stretch between locations, Roški slap and Skradinski buk.

\section{Z-2251. Kalića kuk 1}

$1.7 \pm 0.8 \mathrm{pMC}$

Old tufa barrier, presently 10-15 m above the lake surface. Hard, compact tufa from the top of the barrier.

\section{Z-2252. Kalića kuk 2}

$3.0 \pm 0.8 \mathrm{pMC}$

Hard, very porous tufa from the top of the barrier.

\section{Z-2250. Kalića kuk 3}

Hard, porous tufa from a niche dug in the barrier, $\sim 2 \mathrm{~m}$ above the lake surface.

Comment (D.S.): ${ }^{14} \mathrm{C}$ activity of old, preglacial tufa reflects the contamination with recent carbonates, not the age of the samples.

\section{Z-2323. Manojlovac waterfall}

$75.8 \pm 0.8 \mathrm{pMC}$

Tufa crust on limestone.

\section{Z-2324. Manojlovac water mill}

$75.7 \pm 0.8 \mathrm{pMC}$

Tufa crust on wood.

Comment (D.S.): Samples Z-2323 and -2324 were collected in a dry riverbed. This section of the Krka River was diverted in 1908 into a hydroelectric power plant; hence, the samples reflect prebomb ${ }^{14} \mathrm{C}$ activity.

\section{Varaždinske Toplice series}

Tufa from the thermal Varaždinske Toplice spa $\left(46^{\circ} 10^{\prime} \mathrm{N}, 16^{\circ} 25^{\prime} \mathrm{E}\right)$, Croatia. Collected and submitted 1989 by D. Srdoč, N. Horvatinčić and H. Chafetz.

\section{Z-2147. 1}

$0.7 \pm 0.8 \mathrm{pMC}$

Calcareous deposit on cooling tower walls.

$$
\delta^{13} C=0.4 \%
$$


Z-2149. 2

Tufa from the bottom of Gradišce cave.

\section{Z-2155. 3}

Recent tufa from a spring.

Z-2157. 4

Tufa from an old barrier near Sv. Duh chapel.

\section{Z-2158. 5}

Tufa deposit covering Roman ruins.

$$
\begin{array}{r}
2.9 \pm 0.5 \text { pMC } \\
\delta^{13} C=-1.1 \% \circ \\
0.5 \pm 0.6 \text { pMC } \\
\delta^{13} C=0.8 \% o \\
0.0 \text { pMC } \\
\delta^{13} C=-2.1 \% \circ \\
5.3 \pm 0.4 \text { pMC } \\
\delta^{13} C=0.7 \% \circ
\end{array}
$$

Comment (D.S.): $\delta^{13} \mathrm{C}$ values indicate a non-biogenic origin of the tufa. ${ }^{14} \mathrm{C}$ activity is due to contamination with recent carbonates.

Z-2148. 076-4-2(49-1)

$5730 \pm 110$

Alluvial-proluvial sediment southwest of Šabac $\left(44^{\circ} 47^{\prime} \mathrm{N}, 19^{\circ} 39^{\prime} \mathrm{E}\right)$, Serbia, collected and submitted 1989 by M. Rakić, Geological Institute, Belgrade, for drafting a geological map of Yugoslavia.

\section{Baška series}

Charcoal fragments in clayey sandy gravel near Baška $\left(44^{\circ} 59^{\prime} \mathrm{N}, 14^{\circ} 45^{\prime} \mathrm{E}\right)$, Krk Island, Croatia. Collected and submitted 1989 by L. Marjanac, INA Project, Zagreb.

Comment: Expected age: Middle Pleistocene. Samples were dated to interpret paleofacies.

\section{Z-2150. Baška 1-XXV-6-1}

$5130 \pm 300$

Organic soil, $8 \mathrm{~m}$ asl.

\section{Z-2151. Baška 3-XXIV-6-1}

$25,610 \pm 2640$

Organic soil, $8 \mathrm{~m}$ asl.

\section{Z-2153. Baška 117/2-6}

Organic soil, $10 \mathrm{~m}$ asl.

\section{Z-2152. Karojba}

$98.3 \pm 0.9 \mathrm{pMC}$

Fragment of driftwood near Karojba $\left(45^{\circ} 18^{\prime} \mathrm{N}, 13^{\circ} 49^{\prime} \mathrm{E}\right)$, Istria, west Croatia. Collected 1989 by S. Puškarić and submitted by M. Mihovilović, Istarski boksiti, Rovinj.

\section{Z-2166. Krupa River}

$45.3 \pm 0.7 \mathrm{pMC}$

Tufa covering the bed of the Krupa River $\left(44^{\circ} 11^{\prime} \mathrm{N}, 15^{\circ} 54^{\prime} \mathrm{E}\right)$, Bukovica, south Croatia. Collected and submitted 1989 by D. Srdoč, N. Horvatinčić and H. Chafetz.

\section{Z-2167. Krčić}

$56.0 \pm 0.7 \mathrm{pMC}$

Tufa from Krčić brook waterfall, downstream from Krčić village $\left(44^{\circ} 01^{\prime} \mathrm{N}, 16^{\circ} 18^{\prime} \mathrm{E}\right)$ near Knin, 
south Croatia. Collected and submitted by D. Srdoč and H. Chafetz.

Z-2180. Pag 5-VI-7-K

$>\mathbf{3 7 , 0 0 0}$

Organic soil from Pag Island $\left(44^{\circ} 26^{\prime} \mathrm{N}, 15^{\circ} 04^{\prime} \mathrm{E}\right)$. Collected and submitted 1989 by L. Marjanac.

UNITED STATES

Z-2191. White Bluff

$91.6 \pm 0.8 \mathrm{pMC}$

Recent tufa from White Bluff, Texas. Collected and submitted 1990 by H. Chafetz.

Comment: Sample was dated to determine the initial ${ }^{14} \mathrm{C}$ activity.

\section{CZECHOSLOVAKIA}

\section{Czechoslovakia series}

Tufa samples from central Bohemian Karst and Mt. Male Karpaty, west Slovakia were dated to determine initial ${ }^{14} \mathrm{C}$ activity. Collected and submitted 1987 by N. Horvatinčić and J. Šilar.

\section{Z-2115. Koda}

$99.6 \pm 1.2 \mathrm{pMC}$

Recent tufa covered by aquatic moss (Cratoneurum commutatum) from Koda valley near Srbsko $\left(49^{\circ} 50^{\prime} \mathrm{N}, 14^{\circ} 15^{\prime} \mathrm{E}\right), 320 \mathrm{~m}$ asl.

\section{Z-1979. Koda}

$91.9 \pm 1.3 \mathrm{pMC}$

Recent tufa, $300 \mathrm{~m}$ downstream, at Koda near Srbsko.

$$
\delta^{13} \mathrm{C}=-9.0 \pm 0.4 \%
$$

\section{Z-1976. Cisarska Rokle}

$81.8 \pm 1.2 \mathrm{pMC}$ $\delta^{13} \mathrm{C}=-9.3 \pm 0.4 \% 0$

Recent tufa, $150 \mathrm{~m}$ downstream from a lower eroded cascade at Cisarska Rokle $\left(49^{\circ} 52^{\prime} \mathrm{N}\right.$, $\left.17^{\circ} 02^{\prime} \mathrm{E}\right)$.

\section{Z-1977. Cisarska Rokle}

$89.2 \pm 1.2 \mathrm{pMC}$ $\delta^{13} \mathrm{C}=-7.1 \pm 0.4 \% 0$

Recent tufa, $300 \mathrm{~m}$ downstream from a lower eroded cascade at Cisarska Rokle.

Z-1978. Cisarska Rokle

$46.1 \pm 0.9 \mathrm{pMC}$

Tufa from a lower eroded cascade, on a bank near the waterfall.

\section{Z-1980. Hradište pod Vratnom}

$$
0.1 \pm 0.6 \mathrm{pMC}
$$

Old tufa from a quarry at Hradište pod Vratnom, Mt. Male Karpaty $\left(49^{\circ} 00^{\prime} \mathrm{N}, 17^{\circ} 25^{\prime} \mathrm{E}\right)$.

\section{Z-1981. Sv. Jan pod Skalou 8}

$46.1 \pm 0.9 \mathrm{pMC}$

Dark gray tufa from Sv. Jan pod Skalou $\left(49^{\circ} 53^{\prime} \mathrm{N}, 17^{\circ} 02^{\prime} \mathrm{E}\right)$.

Z-1982. Sv. Jan pod Skalou 9

$57.0 \pm 1.0 \mathrm{pMC}$

Yellowish porous tufa. 
Porous tufa with clay particles.

\section{Z-1984. Sv. Jan pod Skalou 11}

$51.9 \pm 0.9 \mathrm{pMC}$

Yellowish porous tufa, $30 \mathrm{~cm}$ above Sample 10 .

\section{REFERENCES}

Benkö, L., Horvath, F., Horvatincić, N. and Obelić, B. 1989 Radiocarbon and thermoluminescence dating of prehistoric sites in Hungary and Yugoslavia. In Long, A., Kra, R. S. and Srdoc, D., eds., Proceedings of the 13th International ${ }^{14} \mathrm{C}$ Conference. Radiocarbon 31(3): 992-1002.

Degmedžić, J. 1977 The Frankopans and the Sv. Lovro church. In Strbašić, M., ed., Požega, 1227-1977. Zagreb, Grafički zavod Hrvatske: $158-160$ (in Croatian).

Durman, A. and Obelić, B. 1989 Radiocarbon dating of the Vučedol culture complex. In Long, A., Kra, R. S. and Srdoč, D., eds., Proceedings of the 13th International ${ }^{14} \mathrm{C}$ Conference. Radiocarbon 31(3): 1003-1009.

Đurdjević, M. 1987 Barice/Laminci. Ljubljana, Arheološki pregled 1986: 50-51 (in Slovenian).

Horvat, Z. and Mirnik, I. 1977 The Middle Age architecture of the Požega Valley. In Strbašić, M., ed., Požega, 1227-1977. Zagreb, Graficki zavod Hrvatske: 121157. (in Croatian)

Horvath, F. 1982 The Late Neolithic stratum of the Gorsza tell. Archaeologiai Ertesitö 109: 201-222.

1986 Aspects of Late Neolithic changes in the Tisza region. Beri Balogh Adám Múzeum Evkönyve 13: 89-102.

1987 Hádmezövásárhely-Gorsza. A settlement of the Tisza culture. In Tálas, L., Raczky, P., Bökönyi, S., Kalicz, N., Selmeczy, L., Trogmayer, O. and Kelemen, E., eds., The Late Neolithic of the Tisza Region. Budapest-Szolnok: 31-46.

Krajcar Bronić, I., Horvatincić, N., Srdoč, D. and Obelić, B. 1986 On the initial ${ }^{14} \mathrm{C}$ activity in karst aquifers with short mean residence time. In Stuiver, M. and $\mathrm{Kra}, \mathbf{R}$. S., eds., Proceedings of the 12 th International ${ }^{14} \mathrm{C}$ Conference. Radiocarbon 28(2A): 436-440.

1992 Experimental determination of the ${ }^{14} \mathrm{C}$ initial activity of calcareous deposits. In Long, A. and $\mathrm{Kra}, \mathrm{R}$. S., eds., Proceedings of the 14 th International ${ }^{14} \mathrm{C}$ Conference. Radiocarbon, in press.

Langhamer, J. 1966 The chronology of Sv. Lovro church. Požeški zbornik II, Ogranak Matice hrvatske, Slavonska Požega: 149-171 (in Croatian).

Marčenko, E., Srdoč, D., Golubić, S., Pezdič, J. and Head, M. J. 1989 Carbon uptake in aquatic plants deduced from their natural ${ }^{13} \mathrm{C}$ and ${ }^{14} \mathrm{C}$ content. In Long, A., Kra, R. S. and Srdox, D., eds., Proceedings of the 13 th International ${ }^{14} \mathrm{C}$ Conference. Radiocarbon 31(3): 785-794.

Milovanović, B., Stanković, S., Komatina, M., Hadžišehović, M., Župančić, M., Miljević, N., Stepić, R. and Obelić, B. 1989 Isotopic investigation of the Surdulica geothermal system. In Long, A., Kra, R. S. and Srdoc, D., eds., Proceedings of the 13th International ${ }^{14} \mathrm{C}$ Conference. Radiocarbon 31(3): 893-901.

Mook, W. G. 1976 The dissolution-exchange model for dating ground water with ${ }^{14} \mathrm{C}$. Interpretation of environmental isotope and hydrochemical data. Groundwater Hydrology. Vienna, IAEA: 213-225.

Petrović, D. 1984 Sesalačka pećina cave. Beograd, Zbornik radova Instituta za geografiju 31: 9-18 (in Serbian).

Srdoč, D., Breyer, B. and Sliepčević, A. 1971 Rudjer Bošković Institute radiocarbon measurements I. Radiocarbon 13(1): 135-140.

Srdoč, D., Krajcar Bronić, I., Horvatinčić, N. and Obelić, B. 1986a Increase of ${ }^{14} \mathrm{C}$ activity of dissolved inorganic carbon along a river course. In Stuiver, M. and $\mathrm{Kra}, \mathrm{R}$. S., eds, Proceedings of the 12 th International ${ }^{14} \mathrm{C}$ Conference. Radiocarbon 28(2A): 515-521.

Srdoč, D., Obelić, B., Horvatinčić, N., Krajcar Bronić, I., Marčnko, E., Merkt, J., Wong, H. K. and Sliepčević, A. 1986b Radiocarbon dating of lake sediment from two karst lakes in Yugoslavia. In Stuiver, M. and $\mathrm{Kra}, \mathrm{R}$. S., eds, Proceedings of the 12th International ${ }^{14} \mathrm{C}$ Conference. Radiocarbon 28(2A): 495-502.

Srdoč, D., Obelić, B., Horvatinčić, N., Krajcar Bronić, I. and Sliepčević, A. 1984 Rudjer Bošković Institute radiocarbon measurements VIII. Radiocarbon 26(3): 449-460.

1989 Rudjer Bošković Institute radiocarbon measurements XI. Radiocarbon 31(1): 85-98.

Srdoc, D., Obelić, B., Sliepcević, A., Krajcar Bronić, I. and Horvatincić, N. 1987 Rudjer Bošković Institute radiocarbon measurements X. Radiocarbon 29(1): 135-147.

Srdoč, D., Sliepčević, A. Obelić, B. and Horvatinčić, N. 1979 Rudjer Bošković Institute radiocarbon measurements V. Radiocarbon 21(1): 131-137.

Stuiver, M. and Polach, H. A. 1977 Discussion: Reporting of ${ }^{14} \mathrm{C}$ data. Radiocarbon 19(3): 355-363.

Stuiver, M. and Reimer, P. J. 1987 User's guide to the programs CALIB and DISPLAY Rev 2.1: Quaternary Isotope Laboratory, University of Washington. 